\title{
Left common femoral vein thrombosis with lower limb gangrene in a newborn
}

\begin{abstract}
Gangrene in the newborn following deep vein thrombosis is extremely rare and may be congenital and acquired. We report an eleven day term male baby presented in emergency with bluish colouration of left lower limb for two days, which progressed to gangrene on fourth day of admission. The baby was initially treated with low molecular weight heparin followed by warfarin and antimicrobials (cefotaxime and piperacillin). Knee disarticulation was done on the fourth day of admission. On postoperative days, baby was well, and discharged on warfarin therapy. The exact cause for venous thrombosis could not be established. This case is reported because of possible idiopathic gangrene in the newborn with highlights of management.
\end{abstract}

Keywords: newborn, gangrene, deep vein thrombosis
Volume 5 Issue 8 - 2016

Rajniti Prasad,Vinod kumar, OP Mishra, Ashish verma

Department of Pediatrics and radiodiagnosis, Institute of Medical Sciences, India

Correspondence: Rajniti Prasad,Associate professor, Department of Pediatrics, Banaras Hindu University, Varanasi, India, Email rajnitip@gmail.com

Received: July 31, 2016 | Published: December 07, 2016

\section{Introduction}

It is rare to see peripheral limb ischemia and gangrene occurring in the first few days of life. Gangrene may be precipitated by a wide variety of prenatal, natal and postnatal factors that leads to venous occlusion. Other risk factors are sepsis, asphyxia and dehydration. ${ }^{1,2} \mathrm{We}$ describe a healthy newborn presented with left common femoral vein thrombosis with left lower limb gangrene at day eleven of life. There were no risk factors, family history, or any venous catheterisation. On day four of admission, disarticulation at left knee joint was done and the newborn was discharged after ten postoperative days. No cause for venous thrombosis could be established.

\section{Case summary}

An eleven day old male, delivered from gravid three mother by spontaneous vaginal delivery had presented to pediatric emergency with bluish coloration of left toes and lower limb for two days. His birth weight was $3.210 \mathrm{~kg}$. There was no history of perinatal asphyxia, hospitalization, trauma, venous line insertion or umbilical vein/ femoral vein catheterisation. Her mother had no history of diabetes, hypertension, recurrent abortions, rash over face or joint pain. Her previous two deliveries were normal and are alive and well.

On examination, baby was active with stable vitals (HR:142/ min., RR:36/min., Capillary Refill Time $<3 \mathrm{sec}$.). Bilateral radial, right femoral and dorsalis pedis pulses were palpable. Left femoral and dorsalis pedis pulses were non palpable. Left lower limb was bluish in colour and cold to touch. The examination of cardiovascular, chest, abdomen and central nervous system were normal. Over two days, bluish discolouration progressed to gangrene of left lower limb (Figure 1).

His hemogram revealed $\mathrm{Hb}: 10.8 \mathrm{gm} / \mathrm{dl}$, TLC:15,000/mm3, DLC: N76,L20,E3,M1 and Platelet: $140,000 / \mathrm{mm}^{3}$. His renal and liver function tests were normal. There was no evidence of hemolysis in peripheral smear with reticulocyte count less than $0.5 \%$ and negative coomb's test. His c-reactive protein was $0.91 \mathrm{mg} / \mathrm{L}$ (non-significant) and aerobic blood culture was sterile. Prothrombin and activated partial thromboplastin time were normal. Test for anti-cardiolipin antibodies [IgG:6 GPLU/mL(N:<10), IgM MPLU/mL(N:<10) and anti-thrombin III:24.4mg/dL (normal range:19-39 mg/dL) were negative. Serum
protein-C was $77.8 \%$ of normal (deficiency $<$ if less than $60 \%$ ) and protein-S: $91.7 \%$ (deficiency<if less than $70 \%$ ) was within normal limit. Factor V mutation analysis revealed no abnormality. Colour Doppler study of lower limb showed uncompressible common iliac vein with echogenic lumen and no evidence of flow, suggestive of left common iliac vein thrombosis, extending up to superficial femoral vein (Figure 2).

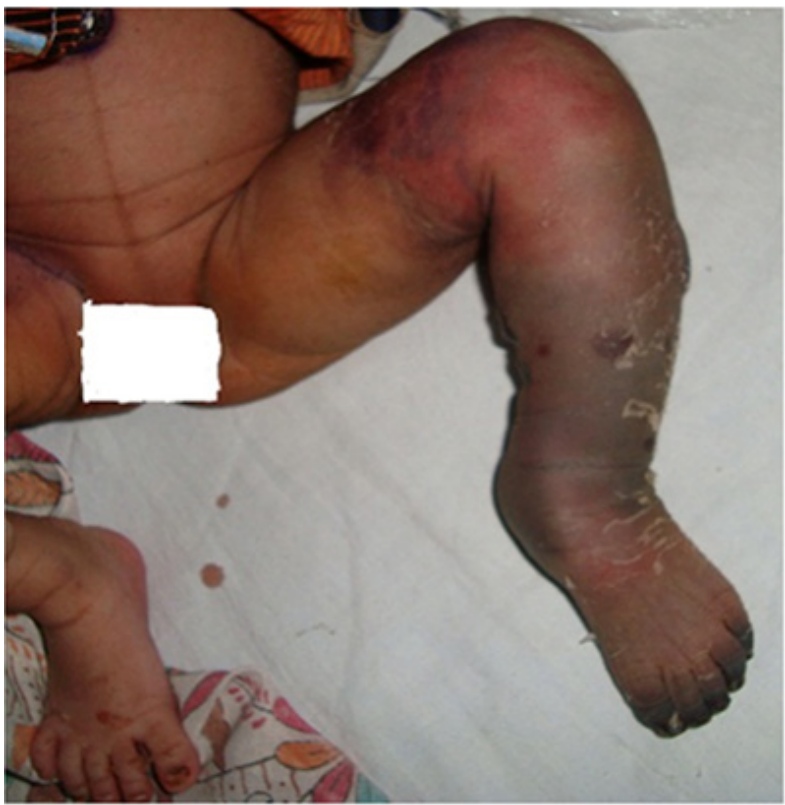

Figure I Gangrene of left lower limb.

\section{Treatment and course in hospital}

At admission, intravenous antibiotics (cefotaxime and piperacillin) and subcutaneous low molecular weight heparin was started. Orthopaedic consultation was done for progressive gangrene of left lower limb on day three of admission and patient was subjected to disarticulation at left knee joint on day four based on extent of gangrene as judged by orthopaedic colleagues. Baby was well in postoperative period and discharged after ten postoperative days. 


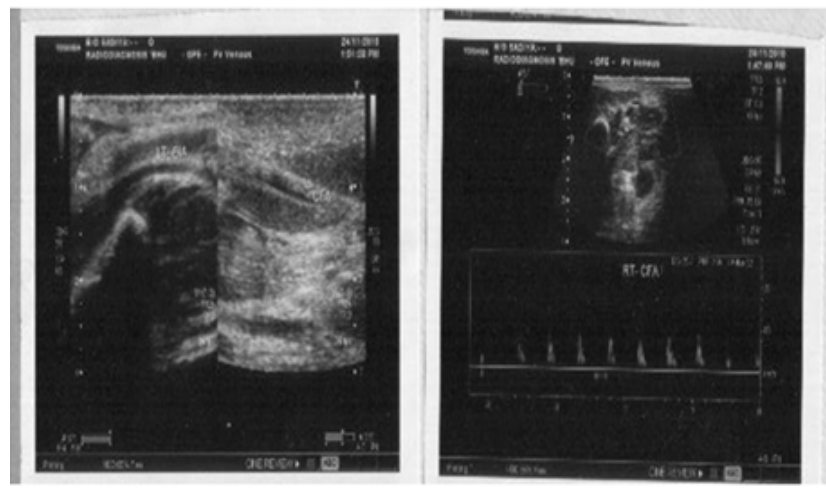

Figure 2 USG showing left common iliac vein thrombosis.

\section{Discussion}

Spontaneous lower limb gangrene with femoral vein thrombosis in a healthy newborn without any risk factor is a rare event. Very few cases have been reported. Although, thromboembolic event is rare in neonates, the peak incidence in the pediatric age group have been reported in neonates and infants less than one year of age. ${ }^{3}$ The relatively higher incidence in neonates as compared to older children may be due to higher hematocrit, and the greater liability of the hemostatic system in neonates due to the generally decreased levels of both coagulation factors and their inhibitors in this age group, except factor VIII (FVIII) and von Willebrand factor (VWF) which are normal or even elevated. ${ }^{4}$

Registries from Canada, and Germany have reported series of cases of neonatal thrombosis. Incidence of clinically significant thrombosis was estimated at 2.4/1,000 admission to newborn intensive care in Canada and 5.1/100,000 births in Germany. ${ }^{5,6}$ The comparable incidence of venous thromboembolic events in the adult population is estimated at 2.5 to $5.0 \%$ of the population. ${ }^{7}$ More than $80 \%$ of thromboembolism in childhood were on a background of a severe preceding illness or other comparable predisposing factors. ${ }^{8}$ The management consist of supportive therapy, anticoagulation, thrombolysis and in some cases surgery. Amputation should be done following appearance of demarcation line as in our patient. ${ }^{9}$

\section{Acknowledgements}

None.

\section{Conflicts of interest}

The authors declare no conflict of interest.

\section{Funding}

None.

\section{References}

1. Ozgenel GY, Akin S, Uysal A, et al. Gangrene of the upper extremity in the newborn. Eur J Plast Surg 23(8):429-431.

2. Braly BD. Neonatal arterial thrombosis and embolism. Surgery. 2000;58(5):869-873.

3. O'Neill JA, Neblett WW, Born ML. Management of major thromboembolic complications of umbilical artery catheters. $J$ Pediatr Surg. 1981;16(6):972-978.

4. Monagle $\mathrm{P}$, Barnes $\mathrm{C}$, Ignjatovic $\mathrm{V}$, et al. Developmental haemostasis:Impact for clinical haemostasis laboratories. Thromb Haemost. 2006;95(2):362-372.

5. Schmidt B, Andrew M. Neonatal thrombosis:report of a prospective Canadian and international registry. Paediatrics. 1995;96(5 Pt 1):939943

6. Nowak-Gottl U, von Kries R, Gobel U. Neonatal symptomatic thromboembolism in Germany:two year survey. Arch Dis Child Fetal Neonatal Ed. 1997;76(3):F163-F167.

7. Carter C, Gent M. The epidemiology of venous thrombosis. In:Colman $\mathrm{R}$, et al. editors. Hemostasis and Thrombosis. Basic Principles and Clinical Practice. Philadelphia JB, Lippincott Company, USA;1982. p. 805-819.

8. Kuhle S, Massicotte P, Chan A, Adams M, et al. Systemic thromboembolism in children:Data from the 1-800-NO- CLOTS Consultation Service. Thromb Haemost. 2004;92(4):722-728.

9. Rea WJ, Wyrick WJ. Necrotizing fasciitis. Ann Surg. 1970;172:957-964. 